\title{
Diacronie
}

Studi di Storia Contemporanea

$\mathrm{N}^{\circ} 18,2 \mid 2014$

Le esposizioni: propaganda e costruzione identitaria

\section{L'Italia alle esposizioni universali del XIX secolo: identità nazionale e strategie comunicative}

\section{Anna Pellegrino}

\section{(2) OpenEdition}

\section{Journals}

Edizione digitale

URL: http://journals.openedition.org/diacronie/1171

DOI: 10.4000/diacronie.1171

ISSN: 2038-0925

Editore

Association culturelle Diacronie

Notizia bibliografica digitale

Anna Pellegrino, «L'Italia alle esposizioni universali del XIX secolo: identità nazionale e strategie comunicative », Diacronie [Online], № 18, 2 | 2014, documento 2, Messo online il 01 juin 2014, consultato il 20 avril 2019. URL : http://journals.openedition.org/diacronie/1171 ; DOI : 10.4000/ diacronie. 1171 


\title{
Diacronie
}

\section{2/}

\section{L'Italia alle esposizioni universali del XIX secolo: identità nazionale e strategie comunicative}

\author{
Anna PELLEGRINO *
}

Questo saggio esamina il modo in cui si afferma e si diffonde nelle esposizioni internazionali della seconda metà del XIX secolo, la presenza di un "prodotto italiano", di uno stile nazionale in alcuni settori specifici, in particolare in alcune lavorazioni specializzate di lusso e di alto artigianato. L'analisi si dipana lungo due direzioni parallele: da una parte l'effettiva presenza degli espositori italiani da un punto di vista quantitativo; dall'altra, il modo in cui i prodotti italiani erano rappresentati sulla stampa illustrata internazionale per alcuni significativi campioni.

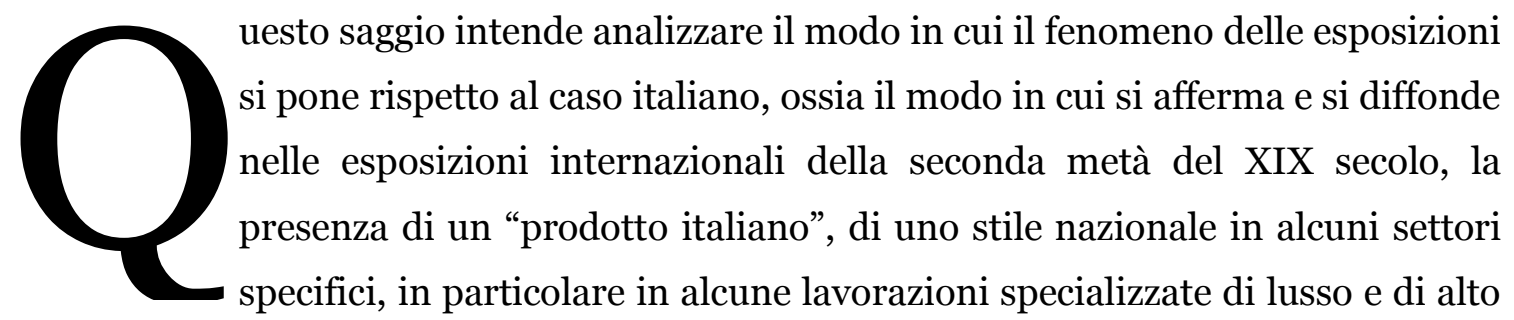
artigianato.

Una componente rilevante dell'affermazione dell'identità nazionale su scala comparativa (lo è tutt'ora in quasi tutti gli eventi di questo tipo) era lo spazio fisico riservato alla singola nazione partecipante. Avere uno spazio adeguato, direttamente proporzionato alla specifica potenza nazionale era un elemento di rilevante interesse per la nazione partecipante. Ancora più importante, e comunque correlato con lo spazio disponibile, era il numero degli espositori ammessi. A differenza degli spazi, 
quest'ultimo indicatore è quantificabile statisticamente in maniera relativamente esatta e costante, per cui mi baserò soprattutto su di esso.

Un ulteriore elemento di affermazione nazionale era offerto dal meccanismo dei premi. I premi, infatti, erano attribuiti ai singoli espositori, ma con procedure che si basavano, dalla composizione delle giurie fino alla redazione delle graduatorie, sulle appartenenze nazionali. Tornare in patria con un alto numero di ricompense (come del resto avviene ancora oggi per le Olimpiadi) era considerato un grande fattore di prestigio sul piano internazionale.

La mia analisi si svolgerà lungo due direzioni in certo modo parallele, ma entrambe necessarie e interconnesse: prenderò in esame sia l'effettiva presenza degli espositori italiani e le tipologie di prodotti, sia il modo in cui essi erano rappresentati sulla stampa illustrata internazionale, almeno per alcuni significativi campioni.

Una delle fonti su cui mi sono basata è costituita dal repertorio iconografico delle quattro maggiori riviste illustrate europee dell'Ottocento, nelle rispettive nazioni di Inghilterra, Francia, Germania e Italia: il «The Illustrated London News», «l'Illustrierte Zeitung», «l'Illustration» e «l'Illustrazione Italiana».

Le quattro riviste analizzate costituiscono un corpus omogeneo, in quanto seguono lo stesso modello editoriale. Le immagini presentate, la loro tipologia, la loro stessa quantità, inoltre, non sono certamente casuali, ma dipendono da precise scelte editoriali. Questo permette di operare delle comparazioni transnazionali su scala abbastanza omogenea. Ho costruito un repertorio che cataloga tutte le immagini concernenti, le dieci maggiori esposizioni universali della seconda metà del XIX secolo evidenziando, dov'era possibile, il riferimento delle illustrazioni stesse a un prodotto o contesto nazionale ben identificabile ${ }^{1}$. Inoltre, sulla base dei dati già noti in letteratura, ho elaborato una tabella che offre un quadro della partecipazione di queste stesse quattro grandi nazioni europee alle dieci esposizioni maggiori del XIX secolo secondo il numero di espositori presenti per ciascun paese, in modo da poter comparare la quantità di materiale iconografico presente sui canali di comunicazione con l'effettiva presenza di espositori all'interno dell'evento. Infine, per arricchire il quadro molto schematico che emerge dalla relazione tra questi due ordini di fattori, ho analizzato una serie di fonti accessorie, che offrono materiali illustrativi ulteriori rispetto a quelli esibiti nelle grandi riviste illustrate, ossia alcuni cataloghi internazionali e le pubblicazioni edite specificatamente nel contesto nazionale.

${ }^{1}$ Le esposizioni sono: Londra 1851; Parigi 1855; Londra 1862; Parigi 1867; Vienna 1873; Filadelfia 1876; Parigi 1878; Parigi 1889; Chicago 1893; Parigi 1900. La tabella è stata costruita sui dati offerti da SCHROEDER-GUDEHUS, Brigitte, RASMUSSEN, Anne, Les fastes du progrès, cit., pp. 59-143. 


\section{Identità nazionale e competizione internazionale alle origini delle esposizioni universali}

Il Primo Maggio del 1851, mentre veniva inaugurata al Crystal Palace "The Great Exhibition of the works of industry of all Nations", il «The Illustrated London News», la prima e più importante rivista illustrata dell'epoca, commentava l'evento con queste parole:

On the 1st of May - a May-day which will long be memorable in the history of our country - the Queen, with all the splendor of her Court - with all the pomp and pageantry of a royal procession - with solemn and rejoicing music - with salvoes of artillery, amid the enthusiastic shouts of the representatives of a hundred different nations, proclaimed the opening of the Palace of Industry. Seldom have such ceremonies been bestowed in such a cause - not the celebration of the close of a long demoralizing war, but the commencement, as we believe and trust, of a new era of peace and good-will.

Into this Palace we are about to enter - to travers day by day its long avenues and galleries - to describe, to explain, to criticize, to illustrate the various contributions of utility and beauty which it is crowded. Here we shall find, side by side, in friendly competition, prepared to exchange lessons in civilising arts, nations which once met only as deadly enemies ${ }^{2}$.

Nel commento del popolarissimo periodico inglese spiccava, come si vede, un elemento dal forte sapore politico, che era stato al centro, seppure in misura e con toni diversi, del discorso di apertura pronunciato dalla regina Vittoria: l'esposizione non era solo un grande evento economico, un punto di arrivo e di svolta nel commercio mondiale, ma era anche l'inizio di una nuova era di pace e di relazioni amichevoli fra i popoli3.

Questo nuovo "modello" inglese di una "pax britannica" estesa in sostanza a tutto il mondo, si contrapponeva nettamente a quanto era avvenuto negli anni immediatamente precedenti sul continente europeo, travagliato dalle guerre fra le nazioni e da quelle interne sul piano sociale.

Tuttavia, il giornale inglese chiariva vieppiù esplicitamente, che questo elemento di pacificazione sociale e politica non andava inteso come un atteggiamento sterilmente

2 The Illustrated London News, vol. XVIII, 482, 3 may 1851, p. 1.

3 AUERBACH, Jeffrey A., The Great Exhibition of 1851. A Nation on Display, NEw HAvenLondon, Yale University Press, 1999, p. 15 et seq. 
irenico: i conflitti armati non andavano cioè semplicemente negati, bensì superati da una "friendly competition" che avrebbe portato al progresso attraverso un proficuo scambio di "lezioni" reciproche sul terreno delle arti civilizzatrici, ovvero delle produzioni industriali capaci di migliorare il gusto e il benessere della popolazione. Un messaggio che esaltava, ovviamente, l'orientamento libero-scambista dell'Inghilterra e il suo necessario corollario, cioè la libera concorrenza, la "competition" sul mercato di cui parlava il «The Illustrated London News»4.

In realtà, fin dall'inizio, la "competition" non fu così "friendly" come voleva dipingerla il periodico londinese. Dall'altra sponda della Manica, in Francia, il periodico parigino gemello, «L'Illustration», guardava con una certa apprensione e con qualche sospetto alla preparazione dell'esposizione, e soprattutto al modo in cui si profilava la sua rappresentazione mediatica da parte dei mezzi di comunicazione, soprattutto da parte della stampa inglese.

«L'Illustration» era stata notevolmente impegnata nella rappresentazione delle esposizioni nazionali parigine che avevano scandito in Francia tutta la prima metà del secolo XIX; in un primo momento quindi aveva seguito l'evento londinese con grande interesse e attenzione, proponendosi di dedicare alla "Exposition universelle" d'oltremanica una serie di supplementi "de la même étendue que la feuille principale".

Molto presto, tuttavia, questo entusiasmo iniziale si ridimensionò nettamente. All'inizio di marzo si criticavano l'organizzazione e i ritardi di Londra5, e successivamente l'interesse si raffreddò ulteriormente, fino al punto che si rinunciò del tutto al progetto di editare una serie speciale di supplementi.

Già fin dai primi mesi dell'anno, da quando cioè il periodico parigino programmava una "copertura" speciale dell'evento, era chiarissimo l'intento e il taglio nazionale con cui intendeva organizzare la propria rappresentazione, e il modo in cui interpretava la "friendly competition":

Les éditeurs de "L'Illustration" publieront comme annexe de ce journal une suite de suppléments dont l'ensemble composera l'histoire illustre de l'exposition de 1851. Les exposants français approuveront cette résolution, inspirée surtout par le désire de représenter leur intérêt spécial dans le concours industriel de toutes les nations. Les immenses préparatifs qui se font à Londres, en vue d'exploiter un événement où les amours-propres nationaux seront en rivalité, nous portent à croire qu'il n'est

$4 \mathrm{Si}$ veda in generale sull'argomento il recente volume di MACLEOD, Christine, Heroes of invention. Technology, liberalism and British identity 1750-1914, Cambridge, Cambridge university press, 2007.

5 L'Illustration, XVII, 419, 8 marzo 1851, riporta in proposito un articolo apparso sul «Journal des débats». 
pas sans utilité d'ouvrir ici une tribune où nos compatriotes soient assurés de trouver justice et protection. La publicité de "L’Illustration", qui s'étend dans tous les pays du monde où les produits de nos arts et de notre industrie sont intéressés à se faire connaître, indique ce recueil aux préférences de nos exposants ${ }^{6}$.

Il giornale, in effetti, riteneva di dover prendere alcune misure difensive nei confronti di quella che presto sarebbe diventata una vera e propria battaglia mediatica per l'affermazione dei prodotti nazionali. Il lessico utilizzato è già di per sé rivelatore di quello che di lì a poco si sarebbe verificato: termini come rivalità, sentimenti nazionali, compatrioti, protezione rivelano chiaramente fin da subito una forte competizione sul piano internazionale.

Nei mesi successivi la rivista francese continuò le sue recriminazioni prendendo di mira la sua gemella inglese «L'Illustrated London News», notando con disappunto lo spazio insufficiente accordato ai prodotti francesi e ironizzando sul "patriotisme anglais" in luogo del necessario "patriotisme universel"7. Quello che si rimproverava agli organizzatori inglesi, era il fatto che nella predisposizione degli spazi espositivi, non solo la più grande quantità era stata attribuita ai produttori inglesi, che del resto fra madrepatria e colonie finivano per essere preponderanti anche come numero ${ }^{8}$; ma che, anche dal punto di vista della collocazione e della posizione nel percorso espositivo, le rappresentanze delle altre nazioni risultavano svantaggiate.

La battaglia mediatica che si sviluppò successivamente, vide «l'Illustration» arrivare alla dichiarazione che per vendicarsi della scarsa attenzione della stampa inglese ai prodotti delle altre nazioni, e quindi anche della Francia, avrebbe accolto sulle sue pagine quasi esclusivamente i prodotti francesi, realizzando quindi il "boicottaggio" di quelli inglesi.

In effetti, «l'Illustration» realizzò il suo boicottaggio dedicando relativamente poco spazio all'evento londinese: pubblicò nel primo semestre 1851 solo una veduta del Crystal Palace e dedicò alla Great Exhibition solo 19 pagine contro le 94 della consorella rivista inglese. Nel corso del secondo semestre l'interesse risultò un poco più

\footnotetext{
6 «Exposition universelle de 1851 à Londres» in L'Tllustration, XVII, 415, 8 febbraio 1851.

7 «Exposition Universelle», in L'Illustration, XVII, 427, 1 maggio 1851 , cit. in TOMASSINI, Luigi, Immagini delle esposizioni universali nelle grandi riviste illustrate europee del XIX secolo in GEPPERT, Alexander C.T., BAIONI, Massimo, «Esposizioni in Europa fra Otto e Novecento. Spazi, organizzazione, rappresentazioni», in Memoria e Ricerca, 17/2004, p.112. ${ }^{8} \mathrm{Su} 14.000$ espositori, 6.861 erano britannici (colonie comprese) di contro ai 1560 della Francia (Algeria compresa); cfr. per questi dati, SCHROEDER-GUDEHUS, Brigitte, RASMUSSEN, Anne, Les fastes du progrès, cit., pp. 58-60.
} 
marcato ma in definitiva la copertura dei prodotti inglesi sul periodico parigino meno di un terzo rispetto ai due terzi dedicati ai prodotti francesi 9 .

Da questo primo scontro mediatico si capisce che fin da subito, nelle esposizioni universali, furono in gioco due elementi: gli spazi e le presenze effettive degli espositori, e spesso, in modo ancora più accentuato, il modo con cui erano questi ultimi erano rappresentati sui primi mezzi di comunicazione di massa dell'epoca, cioè sulla stampa popolare, e in particolare sui giornali illustrati.

Molto prima che le Olimpiadi fossero riprese in chiave moderna, nelle grandi esposizioni universali si affermava il medesimo principio: la cornice era quella di una partecipazione internazionale tendenzialmente universale, basata su principi di pacifica collaborazione; all'interno dell'evento si realizzava però una forte competizione in vista dell'affermazione degli interessi e della stessa complessiva identità nazionale di ciascuna delle nazioni partecipanti. In effetti, in queste manifestazioni progressivamente sempre meno "industriali" e più "universali", entravano in gioco non solo fattori economici, ma anche artistici, culturali, di civiltà.

Le esposizioni universali interessavano all'epoca quantità enormi di persone, fino al culmine di 50 milioni di ingressi all'esposizione parigina del 1900, e raggiungevano un pubblico estremamente vasto ${ }^{10}$; il loro effetto era però ulteriormente amplificato per il fatto che su di esse si innestava una vastissima produzione di rappresentazioni, figurative e scritte (opuscoli, cataloghi ecc.), che venivano a prefigurare, come alcuni

\footnotetext{
9 «Exposition Universelle», in L'Illustration, XVII, 427, 1 maggio 1851.

${ }_{10}$ Fra le opere di carattere generale sulle esposizioni universali, cfr. GREENHALGH, Paul, Ephemeral vistas: the expositions universelles, Great Exhibitions and World's Fairs, 18511939, Manchester, Manchester University Press, 1988; Le livre des expositions universelles 1851-1989, Paris, Edition des arts décoratifs, Hersher, 1983; AIMONE, Linda, OLMO, Carlo, Le Esposizioni Universali 1851-1900. Il progresso in scena, Torino, Allemandi, 1990; SCHROEDER-GUDEHUS, Brigitte, RASMUSSEN, Anne, Les fastes du progrès. Le guide des Expositions universelles 1851-1922, Paris, Flammarion, 1992; BASSIGNANA, Pier Luigi, Le feste popolari del capitalismo. Esposizioni d’industria e coscienza nazionale in Europa, 17981911, Torino Umberto Allemandi \& C., 1997; RYDELL, Robert W., GWINN Nancy E., (a cura di), Fair Representation: World's Fairs and the Modern World, Amsterdam, VU University Press, 1994; WERNER, Peter, Les Expositions universelles au 19ème siècle, spectacles du changement socio-culturel, Bonn-Bad Godesberg, Friedrich-Ebert-Stiftung, 1977; di recente una lettura nuova del fenomeno è avvenuta da parte della storiografia francese, si veda in tal senso CARRÉ, Anne-Laure, CORCY, Marie-Sophie, DEMEULANAERE-DOUYÉRE, Christiane, PÉREZ, Liliane H., Les expositions universelles en France au XIX ${ }^{e}$ siècle. Techniques. Publics. Patrimoines, Paris, CNRS Éditions, 2012; e anche il più recente PÉREZ, Liliane H., DEMEULANAEREDOUYÉRE, Christiane, Les expositions universelles. Les identités au défi de la modernité, Rennes, Presses universitaires de Rennes, 2014; da parte italiana una lettura recente è offerta da MASSIDDA, Luca, Atlante delle grandi esposizioni universali. Storia e geografia del medium espositivo, Milano, Franco Angeli, 2011. Sui pubblici alle esposizioni e in particolare sul pubblico rappresentato dalle classi popolari mi permetto di rimandare al mio recente, PELLEGRINO, Anna, "Macchine come fate". Gli operai italiani alle Esposizioni Universali 1851-1911, Milano, Guerini e Associati, 2011.
} 
storici hanno osservato i primi antenati degli attuali "visual and virtual worlds", così come «i precedenti storici dei processi contemporanei di globalizzazione» ${ }^{11}$.

Le esposizioni rappresentano, in effetti, un medium plurimo e complesso, di cui una parte è realizzata direttamente nell'evento espositivo, un'altra parte è affidata ai nascenti mezzi di comunicazione di massa. Inoltre, per la loro struttura organizzativa (sistema di classificazione per settori e prodotti, giurie, graduatorie e premi differenziati) permettono di stabilire uno sguardo comparativo fra settori merceologici, aree produttive, economie nazionali ma anche fra civiltà, concezioni, stili di vita, mode.

\section{L'Italia alle esposizioni}

Il primo risultato che emerge dalla mia analisi è che la partecipazione italiana alle esposizioni universali è piuttosto debole. È la più bassa, se si considera tutto il periodo nel suo complesso, fra le grandi nazioni europee prese in esame. Il dato naturalmente non può stupire, in quanto è noto che l'industria italiana era relativamente arretrata, e che solo verso la fine del periodo considerato comincia faticosamente a recuperare il ritardo. Inoltre, e soprattutto, influisce sul dato generale anche il fatto che l'Italia, a differenza di Francia e Inghilterra, non riesce ad organizzare nessuna delle grandi esposizioni universali di questo periodo.

L’andamento della presenza italiana è illustrato nel grafico 1.

Grafico 1: Espositori italiani alle maggiori esposizioni universali del XIX secolo in confronto con gli espositori di alcune altre grandi nazioni europee.

${ }_{11}$ Cfr. GEPPERT Alexander, C.T., BAIONI, Massimo, Esposizioni in Europa fra Otto $e$ Novecento, cit., in particolare si veda il saggio introduttivo di GEPPERT, Alexander, Città brevi: storia, storiografia e teoria delle pratiche espositive europee, 1851-200o, cit., pp. 7-18; SLOTERDIJK, Peter, Il palazzo di Cristallo, in ID., Il mondo dentro il capitale, Roma, Meltemi, 2006, pp. 220-228 [ed. or. Im Weltinnenraum des Kapitals, Suhrkamp Verlag, Frankfurt am Main, 2005]; è da notare che l'edizione francese dello stesso volume porta emblematicamente il titolo Le palais de cristal: à l'intérieur du capitalisme planétaire, Paris, Maren Sell Editeurs, 2006. Sulle esposizioni come antecedenti dei processi di comunicazione, si veda inoltre ABRUZZESE, Alberto Lessico della comunicazione, Roma, Meltemi, 2003, pp. 170-183 ; ABRUZZESE, Alberto, BORRELLI, Davide, L'Industria culturale, Tracce e immagini di un privilegio, Roma, Carocci, 2000, pp. 89-92; MATTELART, Armand, Lïnvenzione della comunicazione. Le vie delle idee, Milano, il Saggiatore, 1998, pp. 123-143; FIORENTINO, Giovanni, L'Ottocento fatto immagine:dalla fotografia al cinema, origini della comunicazione di massa, Palermo, Sellerio, 2007, pp. 19-33; e più in generale DE SETA, Cesare, Il secolo della borghesia, Torino, UTET, 2006. 


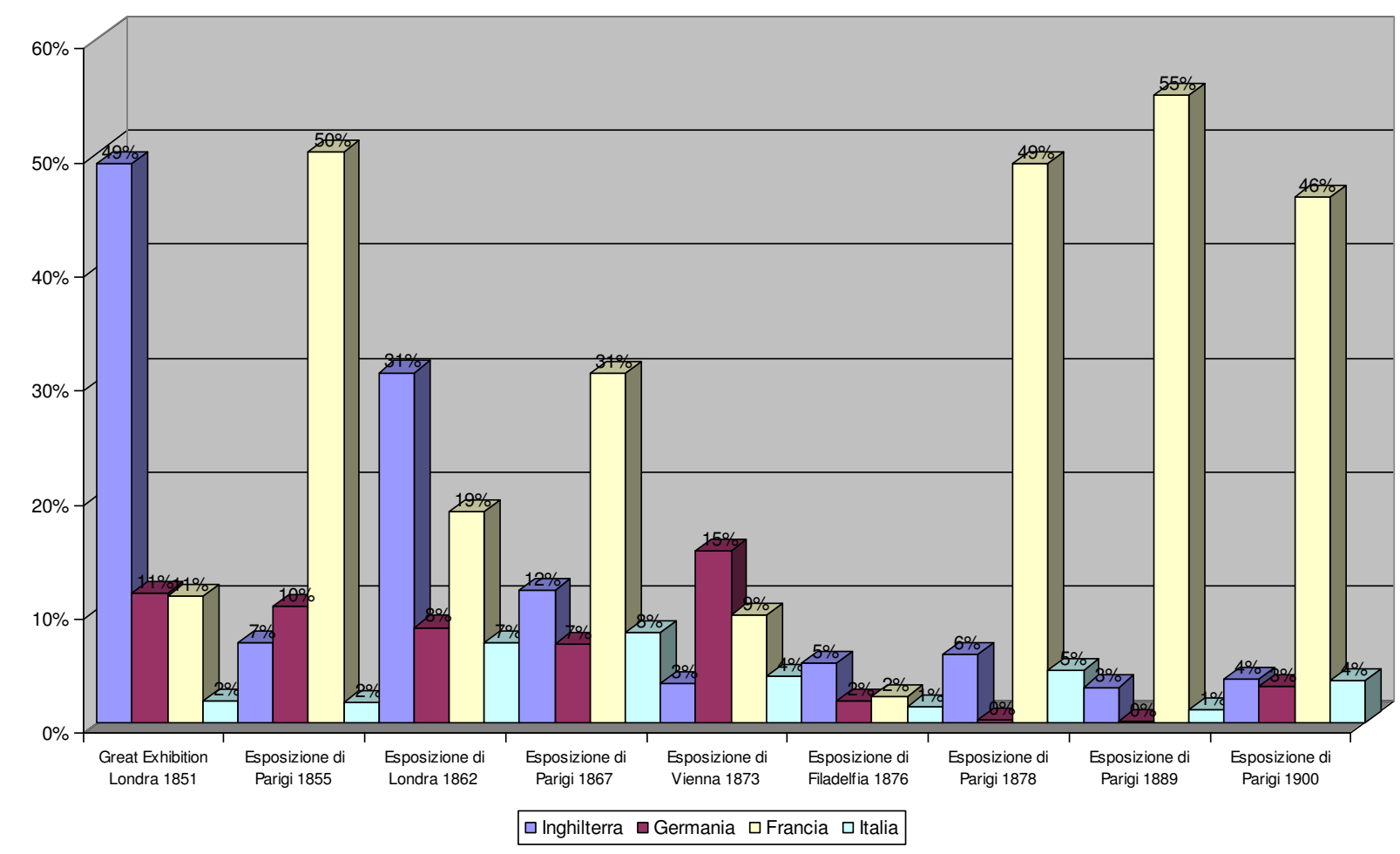

La presenza italiana alle esposizioni varia notevolmente, ma senza mai superare la soglia dell'8\% rispetto al totale degli espositori, raggiunta a Parigi nel 1867. All'esposizione di Londra del 1851 emerge una partecipazione italiana più debole rispetto a quella delle altre grandi nazioni, ed anche diseguale da un punto di vista territoriale, per la frammentazione politica dell'Italia in quel momento. Dei 277 espositori italiani, 57 provengono dallo Stato Pontificio, 97 dal Regno di Sardegna e 123 dalla Toscana.

Nel 1855 a Parigi il numero degli espositori provenienti dall'Italia aumenta sensibilmente e risulta più composita anche la provenienza geografica. I 432 espositori italiani provenivano: 221 dal Regno di Sardegna, 4 dal Regno delle due Sicilie e 207 dal Granducato di Toscana. Nonostante ciò, la percentuale rispetto all'esposizione precedente è addirittura diminuita, dato che a Parigi il numero degli espositori complessivi era assai più alto che a Londra.

Dopo l'Unificazione del paese, la situazione cambia radicalmente. Il nuovo stato italiano già nel 1861 cerca di promuovere una grande esposizione nazionale a Firenze, per inserirsi nel circuito di questo tipo di eventi ${ }^{12}$.

12 Sulla prima esposizione italiana si veda GIUNTINI, Andrea, La prima volta dell'Italia: l'esposizione del 1861 a Firenze, in BIGATTI, Giorgio, ONGER Sergio, Arti Tecnologia Progetto. Le esposizioni d'industria in Italia prima dell'Unita, Milano, Franco Angeli, 2007, pp. 277-290. 
Nel 1862 a Londra gli espositori italiani toccarono i 2.104, ossia quasi quattro volte di più che nel 1855, e soprattutto con una percentuale sul totale degli espositori molto più rilevante che in passato, il $7 \%$.

Questo trend è confermato alla successiva Esposizione di Parigi, dove percentualmente la partecipazione italiana fu la più ampia in tutto il periodo considerato. Parteciparono 4.140 espositori, in assoluto il numero più alto in tutte le esposizioni che ho preso in esame. Questo consentì all'Italia di collocarsi al quarto posto per ordine d'importanza dopo la Francia, l'Inghilterra e l'Impero Ottomano, e addirittura prima della Germania.

Questa situazione che potrebbe apparire singolare se rapportata allo stato delle varie industrie nazionali, era dovuta anche al fatto che in realtà le grandi esposizioni universali, pur se conosciute nel linguaggio comune come esposizioni industriali, non mostravano, com'è noto, solamente lo sviluppo della tecnologia avanzata e della grande industria.

Già dal 1851 il Principe Alberto, principale promotore della Great Exhibition, aveva chiaramente messo al primo posto fra gli obiettivi dell'iniziativa, non tanto quello di esibire i prodotti della grande industria, ma quello di mostrare il livello di perfezionamento del gusto, della moda, delle produzioni delle altre nazioni, per educare al bello la mentalità comune della popolazione inglese e raggiungere per questa via un'elevazione morale (quindi anche obiettivi di carattere politico-sociale) ${ }^{13}$.

In questo modo l'esposizione di Londra del 1851 finì per incontrare una certa ostilità da parte degli industriali inglesi e per dare larghissimo spazio alle cosiddette arti industriali, tendenza che fu largamente confermata nelle esposizioni successive di Parigi e di Londra. In questo contesto anche una industria relativamente arretrata come quella italiana, che però era l'erede di una tradizione artigiana molto antica e gloriosa, poteva avere un suo ruolo di rilievo.

A partire dagli anni Settanta, le cose cambiarono sensibilmente. L'industria tedesca cominciò a svilupparsi a ritmi molto intensi, l'Inghilterra abbandonò progressivamente l'interesse per la diffusione delle arti industriali e in genere per tutto il fenomeno delle esposizioni. Queste ultime finirono per essere destinate sempre più alle innovazioni tecnologiche, all'idea di progresso industriale o a obiettivi di celebrazione della potenza nazionale.

13 Cfr. AUERBACH, Jeffrey A., The Great Exhibition of 1851, cit. p. 15 et seq.; sulla stessa esposizione cfr. anche LEAPMAN, Michael, The World for a Shilling. How the Great Exhibition of 1851 Shaped a Nation, London, Headline, 2001. 
In questa situazione, negli ultimi decenni del secolo, il ruolo dell'Italia iniziò a decrescere su scala comparativa.

Nel 1873 a Vienna, a parte la preminente presenza dell'industria tedesca, la partecipazione italiana era comunque ancora abbastanza forte, con 2.180 espositori, pari ad una percentuale del $4 \%$ sul totale degli espositori.

A Filadelfia nel 1876 la percentuale si può considerare pressappoco dimezzata. In realtà il dato non si riferisce al numero degli espositori ma al numero dei premi ricevuti, visto che l'Italia non partecipò in forma ufficiale.

A Parigi nel 1878 la partecipazione italiana tornò a toccare il 5\% del totale degli espositori, con 2.408 partecipanti.

Tale quota discese drasticamente nel 1889, ma perché, per ragioni politiche, il governo italiano non partecipò ufficialmente all'esposizione che celebrava il centenario della Grande Rivoluzione.

Infine, nel 1900 a Parigi, la partecipazione italiana crebbe nuovamente, fino a 3.130 espositori presenti, per una percentuale del $4 \%$ sul totale degli espositori.

Si tratta di quote molto basse, se confrontate con la partecipazione delle grandi "potenze" industriali dell'epoca, e soprattutto dei paesi dove avevano sede le esposizioni, che naturalmente erano favoriti dal punto di vista organizzativo. Se si confronta però la partecipazione italiana con quella di una potenza industriale di primissimo rango come la Germania, emerge un confronto non altrettanto sfavorevole, dato che, pur facendo salva una prevalenza complessiva della Germania, le rispettive partecipazioni si collocano su una scala perfettamente comparabile: 


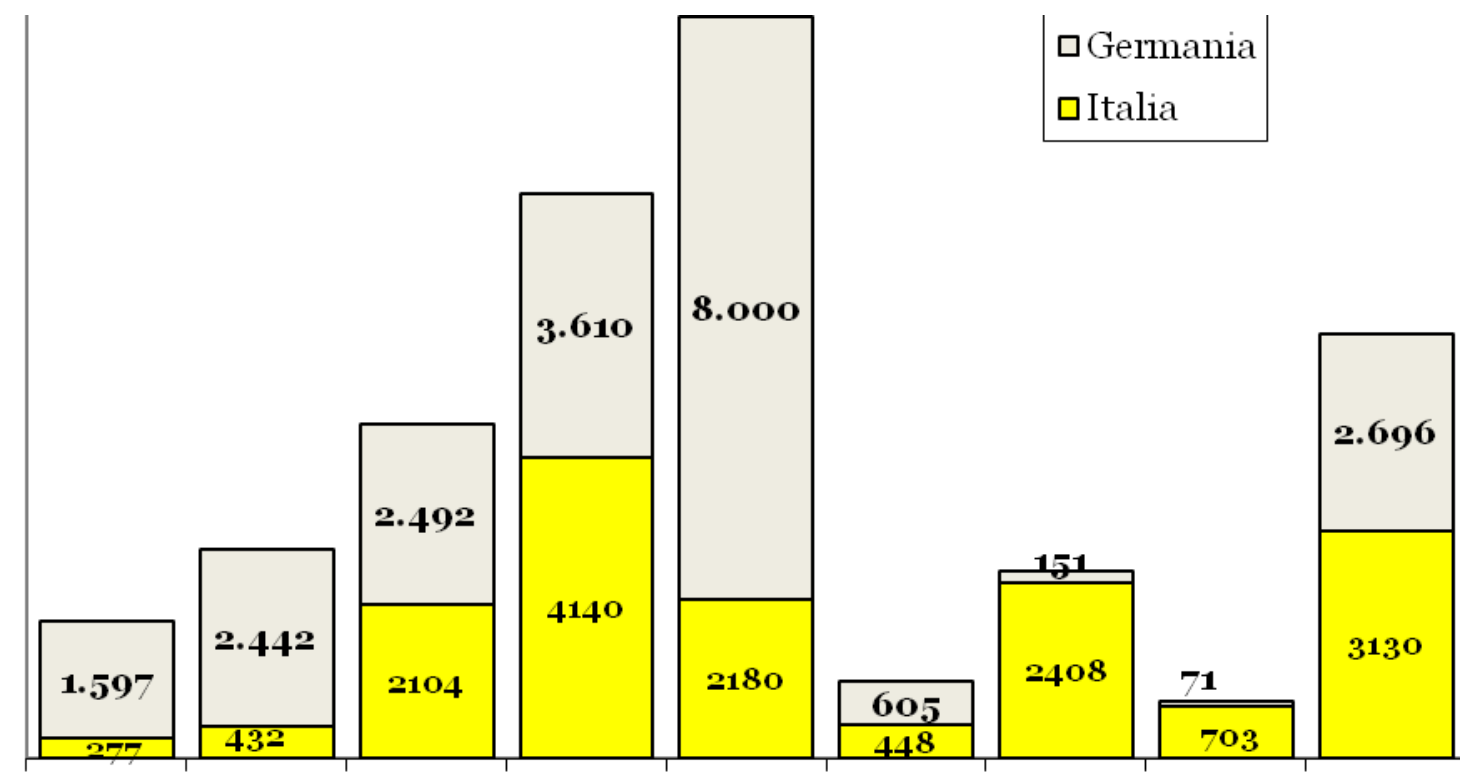

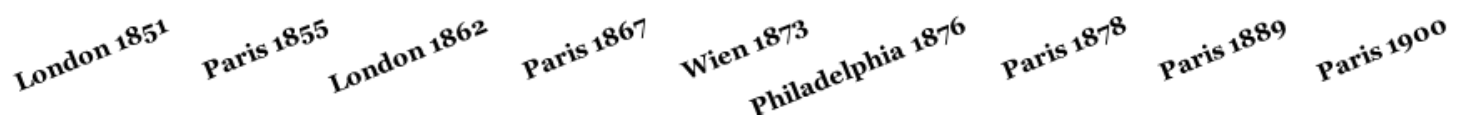

Grafico 2: Espositori italiani e tedeschi nelle principali esposizioni del XIX secolo.

Emerge una presenza italiana che, anche se complessivamente meno rilevante, si rivela abbastanza consistente, con un netto incremento soprattutto dopo l'unificazione del paese e una partecipazione particolarmente elevata alle esposizioni svolte nella vicina Francia (come del resto la massima presenza tedesca si realizza subito dopo la costituzione del Reich all'esposizione tenuta in un paese vicino e alleato come l'Austria).

\section{I prodotti italiani nel circuito mediatico dell'epoca}

Il secondo problema che ci siamo posti all’inizio è quello di verificare, al di là dei dati quantitativi, qual era il livello che raggiungeva l'Italia nelle rappresentazioni e nella percezione diffusa del tempo. Se vi era cioè uno scarto fra la presenza effettiva, il numero assoluto degli espositori e il modo con cui questa partecipazione era valorizzata, sia all'interno del meccanismo organizzativo dell'esposizione, sia nel circuito comunicativo connesso. 
Un primo indice di questa situazione si può rintracciare attraverso il meccanismo dei premi. Le ricompense attribuite all'Italia non seguono in maniera direttamente proporzionale il numero degli espositori presenti, ma a volte sono nettamente inferiori, testimoniando così di una scarsa considerazione dei prodotti italiani da parte delle giurie, peraltro sicuramente influenzate da motivazioni di ordine nazionale nei loro giudizi.

Ad esempio nel 1867 a Parigi l'Italia con 4.140 espositori raccoglie solo 801 ricompense, contro le 1795 della Germania che aveva un numero di espositori minore. Altre volte invece i prodotti italiani ottengono una considerazione migliore, come ad esempio a Vienna nel 1873, quando il rapporto percentuale fra espositori e premi conseguiti è di gran lunga superiore rispetto ad altre nazioni importanti come l'Inghilterra e la Francia e addirittura della stessa Austria che era il paese ospitante ${ }^{14}$.

Il meccanismo dei premi può essere considerato un indicatore assai utile e attendibile della qualità della produzione. Le giurie analizzavano in profondità e con competenza i vari prodotti, la loro natura internazionale garantiva un certo equilibrio nelle valutazioni; in ogni caso, il confronto con altri produttori dello stesso settore portava inevitabilmente a una riconsiderazione in senso migliorativo della propria attività. In alcuni casi, addirittura, questo tipo di meccanismo favoriva cambiamenti radicali nei sistemi produttivi e nella stessa impostazione dell'attività imprenditoriale. Ad esempio all'esposizione di Londra del 1862 il direttore tecnico della manifattura di Doccia, Paolo Lorenzini, nonostante l’indiscutibile successo di pubblico, registrato dalla stampa inglese del tempo e il premio conferito dal Jury, avvertiva privatamente il proprietario della ditta, il marchese Ginori, che in realtà ormai la manifattura era gravemente arretrata nel settore "vitale" della porcellana, carenza controbilanciata solo in parte dalla superiorità dovuta più alla moda e alle oscillazioni del gusto che alla tecnica, nel settore delle maioliche:

Siamo inferiori nella eleganza e varietà dei modelli, nel candore e levigatezza delle paste, $\mathrm{e}$ degli smalti [...], nei sistemi di fabbricazione e di cottura, nei colori, ove l'arte in questi ultimi anni ha fatto dei progressi immensi ${ }^{15}$.

\footnotetext{
${ }_{14}$ Cfr. per tutte queste informazioni, SCHROEDER-GUDEHUS, Brigitte, RASMUSSEN, Anne, Les fastes du progrès, cit., pp. 77 et seq.

${ }_{15}$ Archivio del Museo delle Porcellane di Doccia, Documento n. 2525, in BUTI, Sandra, La Manifattura Ginori. Trasformazioni produttive e condizione operaia (186o-1915), Firenze, Olschki, 1990, p. 24.
} 
Quello che più colpiva i dirigenti della Manifattura fiorentina era che all'estero si andavano diffondendo sistemi innovativi che riducevano il peso delle lavorazioni manuali specializzate a favore di procedimenti meccanici e serializzati, che tuttavia non andavano a scapito della qualità, ma anzi portavano a miglioramenti che, secondo lo stesso Lorenzini, “sono giunti all'estero ad una perfezione sorprendente”. Tutto ciò portava alla necessità di rivedere i propri processi produttivi e anche, almeno in questo caso, la propria "filosofia" imprenditoriale: il marchese Ginori alla fine dovette accettare l'idea che non era più possibile continuare a perseguire una produzione di qualità secondo gli schemi tradizionali e che quindi bisognava imboccare la strada che dall'artigianato tradizionale portava alle nuove "arti industriali":

non solo fino a qui abbiamo fatto degli oggetti d'Arte, ma facevamo gli artisti; d'ora in poi non potremmo più farlo: altri tempi, altri costumi [...] adesso bisognerà di Mecenati ridursi ad esser mercanti e lo faremo: il mestiere non è nuovo per Firenze ${ }^{16}$.

Il meccanismo dei premi era quindi senza dubbio condizionato e influenzato, a sentire i commenti coevi, dalla forza e autorevolezza dei rappresentanti nazionali all'interno delle giurie, ma in complesso, grazie alla grande attenzione cui era esposto e ai controlli incrociati cui era soggetto, rifletteva abbastanza l'effettiva rilevanza "qualitativa” delle varie rappresentanze nazionali.

Dove invece l'Italia fu chiaramente del tutto svantaggiata, e talora quasi del tutto esclusa, non riuscendo a raggiungere quasi nessuna "visibilità" pubblica, fu proprio sul terreno della diffusione mediatica attraverso la stampa. Il meccanismo inaugurato nel 1851, di una concorrenza che si traduceva in una vera e propria "battaglia" mediatica per l'affermazione dei prodotti nazionali, fra l'«Illustrated London News» e «L'Illustration» (riviste, va detto, che avevano una grande diffusione non solo nelle rispettive nazioni, ma toccavano un pubblico molto più ampio su scala mondiale) finiva per mettere in assoluto subordine la partecipazione delle nazioni "minori”. Dalla tabella che segue emerge il quadro della presenza dell'Italia (e dei prodotti italiani) alle dieci maggiori esposizioni della seconda metà del XIX secolo (tab. 3) ${ }^{17}$.

${ }^{16}$ Ibidem, p. 31. Sul tema mi permetto di rinviare anche al mio recente, PELLEGRINO, Anna, Continuité et innovation dans la fabrication de la céramique à Florence entre XIX et XX siècles, actes du XXVII Congrès Préhistorique de France (Bordeaux 1-5 Juin 201O), "Transition, ruptures et continuité en Préhistoire”, Tours, Les Éditions La Simarre, 2013, pp. 113-119.

${ }_{17}$ La tabella è stata costruita sulla base di dati che comprende tutte le illustrazioni apparse sulle quattro riviste sulle pagine delle suddette riviste di Francia, Germania, Inghilterra e Italia in occasioni delle 10 esposizioni maggiori del XIX secolo già enumerate nella nota 12. La banca dati 
Tab. 3: Immagini relative all'Italia nelle maggiori riviste illustrate europee 1851-190o.

\begin{tabular}{|c|c|c|c|c|}
\hline Rivista & Anno & Pagina & $\begin{array}{l}\text { Sede } \\
\text { espositiva }\end{array}$ & Didascalia \\
\hline The Illustrated London News & 1851 & 129 & Londra & Table-top, mosaic (Barberi) \\
\hline The Illustrated London News & 1851 & 132 & Londra & Cabinet (Barbetti) \\
\hline The Illustrated London News & 1851 & 32 & Londra & Psyche (Freccia) \\
\hline The Illustrated London News & 1851 & 32 & Londra & Paolo and Francesca (Munro) \\
\hline The Illustrated London News & 1851 & 32 & Londra & Picture frame, carved (Barbetti) \\
\hline The Illustrated London News & 1851 & 32 & Londra & Slave, Veiled (Monti) \\
\hline The Illustrated London News & 1851 & 522 & Londra & Gratitude (Benzoni) \\
\hline The Illustrated London News & 1851 & 603 & Londra & Dish, silver (Angeli) \\
\hline The Illustrated London News & 1851 & 522 & Londra & Fidelity (Benzoni) \\
\hline The Illustrated London News & 1851 & 252 & Londra & Cannabic decoration (Albano) \\
\hline The Illustrated London News & 1851 & 308 & Londra & Cannabic centre piece (Albano) \\
\hline The Illustrated London News & 1851 & 300 & Londra & Cannabic panels (Albano) \\
\hline The Illustrated London News & 1851 & 472 & Londra & Caractacus (Panormo) \\
\hline The Illustrated London News & 1851 & 362 & Londra & Rosamunda (sculpture) \\
\hline The Illustrated London News & 1862 & 441 & Londra & Italian Court, The, \\
\hline The Illustrated London News & 1862 & 90 & Londra & L'Innominato, \\
\hline Die Illustrierte Zeitung & 1873 & 408 & Vienna & Italienische Abteilung \\
\hline Illustrazione italiana & 1876 & 60 & Filadelfia & Sezione italiana \\
\hline Illustrazione italiana & 1876 & 92 & Filadelfia & Sezione italiana \\
\hline Illustrazione italiana & 1876 & 236 & Filadelfia & Sezione italiana \\
\hline Illustrazione italiana & 1876 & 476 & Filadelfia & Sezione italiana \\
\hline Illustrazione italiana & 1876 & 252 & Filadelfia & Le terre cotte \\
\hline Illustrazione italiana & 1878 & 393 & Parigi & Interno della sezione italiana \\
\hline Illustrazione italiana & 1878 & 233 & Parigi & Facciata della sezione italiana \\
\hline Illustrazione italiana & 1878 & 124 & Parigi & I lavori della sezione italiana \\
\hline The Illustrated London News & 1878 & 588 & Parigi & Italian facade, Champ de Mars \\
\hline Illustrazione italiana & 1878 & 409 & Parigi & $\begin{array}{l}\text { Banchetto offerto dagli italiani al duca } \\
\text { d'Aosta }\end{array}$ \\
\hline Illustrazione italiana & 1878 & 196 & Parigi & Interno della esposizione artistica italiana \\
\hline Illustrazione italiana & 1878 & 360 & Parigi & Barcagli. L'aurora nella vita \\
\hline Illustrazione italiana & 1878 & 304 & Parigi & Caroni (Emanuele). L'africana \\
\hline Illustrazione italiana & 1878 & 344 & Parigi & Emanueli (Giovanni). Il pifferaio \\
\hline
\end{tabular}

è il prodotto di una ricerca realizzata da un gruppo di lavoro dell'Università di Firenze costituitosi nel 2001 sul tema delle guide per i viaggiatori e l'immagine dell'Italia nelle riviste illustrate europee, di cui ho fatto parte, sotto la direzione scientifica del prof. Luigi Tomassini, che qui ringrazio per avermi consentito la piena disponibilità della banca dati. 


\begin{tabular}{|c|c|c|c|c|}
\hline Illustrazione italiana & 1878 & 329 & Parigi & Malfatti (A.). Lacci d'amore \\
\hline Illustrazione italiana & 1878 & 340 & Parigi & Monteverde (Giulio). L'architettura \\
\hline Illustrazione italiana & 1878 & 289 & Parigi & $\begin{array}{l}\text { Monteverde (Giulio). Monumento al conte } \\
\text { Massari }\end{array}$ \\
\hline Illustrazione italiana & 1878 & 88 & Parigi & La sezione italiana \\
\hline L'Illustration & 1878 & 420 & Parigi & Façade de la section italienne \\
\hline Die Illustrierte Zeitung & 1878 & 290 & Parigi & Italienische Skulptur: Die Schwimmerin \\
\hline L'Illustration & 1878 & 272 & Parigi & $\begin{array}{l}\text { L'exposition de M. Domange-Rollin: dame } \\
\text { et damoiselle florentines du seizième } \\
\text { siècle, bustes en bronze de m. Faure de } \\
\text { Brousse }\end{array}$ \\
\hline Illustrazione italiana & 1889 & 236 & Parigi & Padiglione della repubblica di san Marino \\
\hline Illustrazione italiana & 1889 & 176 & Parigi & Sezione italiana agricola \\
\hline Illustrazione italiana & 1889 & 113 & Parigi & Facciata della sezione italiana \\
\hline Illustrazione italiana & 1893 & 292 & Chicago & La sezione italiana \\
\hline Illustrazione italiana & 1893 & 220 & Chicago & La sezione italiana \\
\hline Illustrazione italiana & 1900 & 1 & Parigi & $\begin{array}{l}\text { Il presidente Loubet visita il padiglione } \\
\text { italiano }\end{array}$ \\
\hline Illustrazione italiana & 1900 & 17 & Parigi & $\begin{array}{l}\text { Il presidente Loubet visita il padiglione } \\
\text { italiano }\end{array}$ \\
\hline Illustrazione italiana & 1900 & 10 & Parigi & Sala della sezione italiana di pittura \\
\hline Illustrazione italiana & 1900 & 29 & Parigi & Sezione italiana dell'industria serica \\
\hline Illustrazione italiana & 1900 & 80 & Parigi & Sezione italiana di scultura \\
\hline Illustrazione italiana & 1900 & 265 & Parigi & La danza italiana al palazzo della danza \\
\hline
\end{tabular}

Su un corpus di 3.422 immagini, le illustrazioni riguardanti l'Italia e i suoi prodotti, nei cinquant'anni di esposizioni del XIX secolo, si aggirano attorno ad una cinquantina, pari a meno del 2\% del totale, a fronte di una presenza degli espositori che mediamente si attestava sul doppio o il triplo, con punte attorno all'8\%. Questo dato testimonia chiaramente che l'Italia è assolutamente sottorappresentata in questo tipo di canale mediatico.

Lo scarto diventa ancora più evidente se si considera che più della metà di queste immagini erano apparse sull'«Illustrazione Italiana» e che quasi un terzo (15) erano invece apparse sull' «Illustrated London News» del 1851. In occasione della «Great Exhibition», infatti, il giornale londinese si era mostrato particolarmente aperto, anche per il desiderio di valorizzare la dimensione effettivamente "globale" della partecipazione delle diverse nazioni, verso le produzioni dei vari stati italiani che avevano inviato propri espositori. Gli oggetti privilegiati furono in quell'occasione prodotti di alto artigianato, dipinti e sculture di autori contemporanei, prodotti al 
confine fra artigianato e arte. A questo settore, legato cioè alla diffusione del gusto e del bello ma con riferimento ad un ambito di minor pregio e rivolto a soluzioni più economiche, si possono riferire anche le tre immagini dedicate alla produzione di « wall decorations in Cannabic » prodotte dall'espositore B. Albano (in pratica, un materiale per decorazioni architettoniche composto di gesso e canapa, secondo una formula inventata da Albano stesso, e già noto in Inghilterra per essere stato usato per le decorazioni del teatro del Covent-Garden) ${ }^{18}$.

Il caso dell'«Illustrated London News» del 1851 è tuttavia una eccezione. Nella medesima occasione, l'altro grande giornale illustrato internazionale, «L'Illustration», ignorava del tutto la produzione italiana.

Questo disinteresse aumentò negli anni successivi e coinvolse lo stesso «The Illustrated London News», che dedicò in occasione della successiva esposizione londinese del 1862, solo due immagini all'Italia, e una sola in occasione dell'esposizione parigina del 1878.

In definitiva, quindi, la presenza italiana è sottodimensionata nel circuito comunicativo internazionale anche perché fra le quattro riviste analizzate, «l'Illustrazione Italiana» è quella che nasce più tardi e ha una circolazione più limitata.

Per quanto concerne invece la tipologia dei prodotti, emerge una certa visibilità degli oggetti di alto artigianato, spesso in materiali preziosi, o addirittura di vere e proprie opere d'arte ${ }^{19}$.

Progressivamente, la presenza dell'artigianato di lusso e di qualità sembra cedere il passo alle sculture e alla pittura, oppure a vedute di carattere generale e cerimoniale, con una certa presenza anche delle attività e produzioni agricole.

Nel complesso, anche quando entra in campo «l'Illustrazione Italiana», che indubbiamente contribuisce a innalzare il livello di attenzione verso il nostro paese, non pare sia proposta sul palcoscenico internazionale un'immagine dell'Italia come nazione industrialmente o tecnologicamente avanzata; si punta molto più sul gusto, sullo stile, sulla tradizione delle arti.

\footnotetext{
${ }_{18}$ Come recita la didascalia "Cannabic": «is the name of a new preparation from hemp, intended to supply the place of papier maché and corton pierre, invented and patented by M. Albano. Whilst, perhaps, it has not quite the softness of surface of the former named materials, it has the advantage of great durability and of quickly drying. It was first used by M. Albano in the decoration of Covent-garden Theatre». Nonostante questo uso inglese, come testimonia Andrew Ure (A dictionary of arts, manufactures and mines containing a clear exposition of their principles and practice, vol. 2, p. 612) si trattava di un prodotto «of Italian origin, and in Italy it has been employed for panels, frames, and centres».

$19 \mathrm{Si}$ veda sull'argomento il recentissimo volume di COLOMBO, Paolo, Le Esposizioni Universali. I mestieri d'arte sulla scena del mondo, Venezia, Marsilio, 2012.
} 
Si tratta di una scelta significativa, in altri casi, come ad esempio all'esposizione internazionale di Milano del 1906, il ruolo della tecnologia e dell'industria italiana nel circuito mediatico dell'epoca risultò assai più accentuato. Questa tendenza è confermata dall'analisi di altre tipologie di fonti.

Accanto alle grandi riviste illustrate, che si rivolgevano a un pubblico generalista, e in maniera non specializzata (è vero che in occasione della Great Exhibition del 1851, l'«Illustrated London News» aveva offerto una larghissima copertura all'evento e produceva un apposito supplemento stampato all'interno del Crystal Palace, ma in ogni caso non era mai completamente dedicato all'esposizione), le esposizioni dettero luogo alla produzione di una vasta messe di pubblicazioni specializzate. Sono fonti su cui è estremamente difficile condurre un'analisi sistematica dato che si tratta di materiali molto dispersi e diversi fra loro; ma ho proceduto comunque, per exempla, ad esaminarne alcuni. L'analisi conferma, seppure con misure a volte diverse, l'impressione già ricavata dall'esame delle riviste.

Se prendiamo ad esempio in esame il catalogo pubblicato in Germania sull'esposizione londinese del 1862, Illustrirter Katalog der Londoner Industrier Austellung von 1862 (Lipsing F. A Brockhaus), emerge che sulle 446 pagine complessive dei due volumi, solo 5 sono dedicate a prodotti italiani e precisamente a prodotti di alto artigianato, come "porcellane robbiane" o mobili intagliati fiorentini e milanesi (Barbetti e Ripamonti) fino ai gioielli romani di Castellani. Il The Illustraded Catalogue of The Universal Exhibition published whith the art journal (LondonNewYork, virtue and Co, 1867), pubblicato in occasione dell'esposizione parigina del 1867, su 331 pagine ne dedica un numero un po' superiore, 24 per la precisione, a prodotti italiani; i soggetti sono sempre più o meno simili : sculture milanesi, mobili e intagli fiorentini (Gajani e Frullini), collezioni di porcellane Richard Ginori.

Questa visibilità riservata solo a questa tipologia di oggetti sottolineata criticamente anche da alcuni autorevoli osservatori italiani, rifletteva in realtà l'effettiva caratterizzazione dei prodotti degli espositori italiani come prodotti legati al lusso e alla moda $^{20}$. All'esposizione di Parigi nel 1878 il Conte Carlo Demetrio Finoicchietti dichiarava :

A chiunque percorreva la sezione italiana dei mobili non era dato di vedere un oggetto il cui carattere utile e pratico indicasse i bisogni assoluti di coloro per quali era stato fabbricato. Infine se, alla mostra di Parigi si fosse dovuto dedurre quale

20 Si veda sull'argomento ONGER, Sergio, PARZIALE, Lavinia, «La moda italiana alle esposizioni nella seconda metà dell'Ottocento», in Annali di Storia dell'impresa, 19/2008, Venezia, Marsilio, 2007, pp. 15-47. 
era il corredo abituale delle abitazioni in Italia, avrebbe dovuto indursi, o che i nostri ebanisti e tappezzieri non sanno fabbricare mobili comuni alla portata di qualunque cittadino, oppure che l'Italia è una nazione talmente ricca da potersi permettere per i suoi usi giornalieri soltanto mobili intagliati e riccamente intarsiati, tavole di mosaico e via discorrendo ${ }^{21}$.

\section{La stampa nazionale}

Esiste in definitiva un evidente e comprensibile décalage dell'immagine proposta. Quando l'Italia è presentata in un contesto internazionale, su riviste inglesi, francesi o tedesche, in cui il contesto industriale è indubbiamente più avanzato, l'accento viene posto soprattutto sullo stile, sul gusto, sull'arte, in cui l'Italia tradizionalmente eccelle, in ambito nazionale, invece, si cerca di valorizzare il limitato progresso industriale della nazione, specie negli anni del cosiddetto "decollo industriale".

Questo scarto fra un'immagine "nazionale" e una "internazionale" emerge in particolare nell'analisi delle pubblicazioni edite specificatamente in un contesto nazionale.

Esistono pubblicazioni monografiche interamente dedicate non all'esposizione in sé, ma ai prodotti italiani presentati all'esposizione. Per esempio dal catalogo L'Italia alla Esposizione Universale di Parigi del 1867. Rassegna critica descrittiva illustrata (Firenze, Le Monnier, 1868), emerge pur sempre una notevole quantità d'illustrazioni relative ad opere d'arte, con opere dei maggiori pittori e scultori italiani dell'epoca, come ad esempio Giovanni Dupré, Vincenzo Vela, Domenico Morelli e Stefano Ussi. Una presenza significativa è riservata alle "arti applicate all'industria", attraverso oggetti come le già citate porcellane di Doccia e i vetri di Murano. Erano presenti però, a differenza di quanto accadeva nelle riviste generalistiche, anche prodotti considerati ad alto contenuto tecnologico, come strumenti ottici, scientifici, fotografici. Una qualche presenza era riservata anche alle produzioni manifatturiere.

Talora tutti questi aspetti potevano anche intrecciarsi: una sintesi esemplare di quest'attenzione composita e incrociata a un largo arco di attività produttive, che andava dagli artisti veri e propri fino alle lavorazioni più umili, era sintetizzata in una riproduzione del quadro a olio di Seitz che rappresentava in forma artistica una immagine di donne impegnate nella lavorazione della seta, ma voleva illustrare, nel

${ }^{21}$ FINOCCHIETTI, Demetrio Carlo, Mobili di lusso italiani e mosaici fiorentini, Veneti e Romani, in Esposizione Universale del 1878 in Parigi, Relazione dei giurati italiani, classi XVII e XVIII, Roma Eredi Botta, 1879 
contempo, la novità del metodo di trattura inventato da un ingegnere del settore (Keller).

Anche i cataloghi illustrati di Sonzogno pubblicati appositamente in occasione delle maggiori esposizioni e legittimati dalle stesse commissioni internazionali offrivano un'immagine del tutto analoga ${ }^{22}$. Le immagini concernenti l'Italia in questo tipo di pubblicazioni non erano esclusive, ma erano comunque prevalenti. Anche i testi che accompagnavano le illustrazioni erano solitamente assai celebrativi. Le tipologie di prodotti, andavano dai legni intarsiati e dai merletti di Burano "che si credevano del tutto decaduti" fino alle attività manifatturiere e in particolare all'industria serica, in pieno sviluppo e in grado, secondo il punto di vista "nazionale" di queste fonti, di insidiare la tradizionale egemonia francese nel settore.

Ł̀ come se la partecipazione negata dal circuito mediatico internazionale fosse recuperata all'interno del paese da questa produzione ricchissima e apertamente tagliata secondo un'ottica "nazionale" per valorizzare le produzioni italiane.

In definitiva la partecipazione alle grandi esposizioni, dava luogo in Italia a un circuito nazionale di medializzazione che in qualche modo, sia pure in senso inverso, ripeteva lo stesso meccanismo di inclusione-esclusione su base nazionale che caratterizzava la rappresentazione della presenza dei vari ambiti produttivi nazionali sulle grandi riviste illustrate: si facevano cioè vedere soprattutto i prodotti italiani.

Mentre la partecipazione alle esposizioni non riusciva a garantire all'Italia un ruolo, se non del tutto marginale, all'interno del circuito mediatico internazionale, per contro sul piano interno riusciva ad avere un ruolo di legittimazione importante.

Era cioè essenziale per l'ambiente produttivo italiano spendere la propria presenza alle esposizioni internazionali sul piano interno (si pensi al ruolo dei premi conseguiti alle esposizioni nelle pubblicità dei prodotti dell'epoca) dove questa partecipazione era vista come legittimazione e garanzia della qualità della produzione nazionale.

In conclusione l'Italia giunge assai tardi e con un successo limitato a realizzare una strategia comunicativa per i propri prodotti in quello che poteva essere considerato il canale principale di comunicazione dell'epoca, cioè la stampa e specificamente quella illustrata o specializzata sul tema delle esposizioni.

${ }^{22} \mathrm{Si}$ veda ad esempio, L'Esposizione di Parigi del 1878 illustrata, Milano, Sonzogno, 1878-1879; oppure L'esposizione universale del 1867 illustrata, pubblicazione internazionale autorizzata dalla commissione imperiale dell'esposizione, Milano-Firenze-Venezia, Edoardo Sonzogno, 1867; L'Esposizione Universale di Vienna del 1873 illustrata, Milano, Edoardo Sonzogno, [s.d.]. Sulle esposizioni italiane si veda il Catalogo ufficiale della Esposizione nazionale del 1881 in Milano: belle arti, Milano, E. Sonzogno, 1881; o la Guida del visitatore alla Esposizione industriale italiana del 1881 in Milano: sola pubblicazione autorizzata e compilata sotto la sorveglianza del Comitato esecutivo dell'Esposizione industriale, Milano, Sonzogno, 1881; e ancora il settimanale, «L'esposizione illustrata di Milano». 
Sono al contrario le esposizioni stesse, in quanto evento mediatico, a permettere una presenza apprezzabile degli espositori italiani e anche un grado di legittimazione abbastanza elevato da parte delle giurie. Le esposizioni, con il loro meccanismo di premi e di giurie organizzate in modo da garantire un certo equilibrio nel confronto fra le nazioni partecipanti, forniscono un forte impulso all'affermazione di un'identità nazionale italiana, difficile da far valere nel circuito comunicativo internazionale, ma indubbiamente più facile da manifestare e valorizzare sul piano interno. 


\section{* L'autore}

Anna Pellegrino, assegnista di ricerca Senior presso l'Università degli studi di Padova, è dottore di ricerca dell'Istituto Universitario Europeo (2004). Ha conseguito il DEA in Histoire et civilisation presso L'EHESS di Parigi (1999). Dal 2008 è chercheur associé al Conservatoire National des Arts et Métiers di Parigi, e dal 2010 all'Université Paris Diderot 7. Si occupa di storia delle culture del lavoro nella società industriale, di associazionismo operaio e popolare fra Otto e Novecento e di esposizioni universali fra XIX e XX secolo. Fra le sue pubblicazioni Macchine come fate. Gli operai italiani alle esposizioni universali 1851-1911, Milano, Guerini e Associati, 2011; La citta più artigiana d'Italia. Firenze 1861-1929, Milano, Franco Angeli, 2012, e diversi saggi in riviste italiane e straniere.

URL: < http://www.studistorici.com/progett/autori/\#Pellegrino >

\section{Per citare questo articolo:}

PELLEGRINO, Anna, "L'Italia alle esposizioni universali del XIX secolo : identità nazionale e strategie comunicative», Diacronie. Studi di Storia Contemporanea : Le esposizioni: propaganda e costruzione identitaria, 29/6/2014,

URL: < http://www.studistorici.com/2014/06/29/pellegrino_numero_18/ >

Diacronie Studi di Storia Contemporanea 3 www.diacronie.it

Risorsa digitale indipendente a carattere storiografico. Uscita trimestrale.

redazione.diacronie@hotmail.it

Comitato di redazione: Jacopo Bassi - Luca Bufarale - Elisa Grandi - Deborah Paci - Fausto Pietrancosta - Matteo Tomasoni - Luca Zuccolo

iritti: gli articoli di Diacronie. Studi di Storia Contemporanea sono pubblicati sotto licenza Creative Commons 2.5 Possono essere riprodotti a patto di non modificarne i contenuti e di non usarli per fini commerciali. La citazione di estratti è comunque sempre autorizzata, nei limiti previsti dalla legge. 